\title{
Microsporidioses et cholangiopathies associées à l'infection VIH
}

Depuis la première description de cholécystite alithiasique chez un patient ayant le SIDA en 1983 [1], des anomalics des voies biliaires ont été rapportécs dans environ une centainc de cas chez des patients infectés par le virus de l'immunodéficience humaine (VIH) $[2,3]$. Dans une étude rétrospective conccrnant 15 patients, nous avons défini les données diagnostiques, l'évolution ct l'étiologie de ces cholangiopathies du SIDA [4]. Dans unc étude prospective, nous nous sommes attaché à préciser l'étiologie des cholangiopathies associées à l'infection VIH pour lesquclles aucunc causc n'avait été jusqu'à présent trouvée. Cela nous a permis de mettre cn évidence qu'elles étaient fréquemment associćcs à une infection biliaire à microsporidies.

I a cholangiopathie était une complication rćvćlant le SIDA ou l'infection VIH chez quatre de nos 15 patients (27 \%) alors quc les autres étaient déjà classćs dans le groupc IV (C1, C2 ou D) définissant le SIDA. Lc chiffre moycn de CD4 était de $80 / \mathrm{mm}^{3}$ (4 à 297) et l'antigénémie p24 préscnte chez $71 \%$ des patients. Douze d'entre eux (80 \%) étaient homosexuels. Lcs éléments diagnostiques principaux étaient des douleurs abdominales (73\%), une cholestase anictérique $(100 \%)$, une cryptosporidiose intestinale $(80 \%)$ ct des anomalies de l'échographie abdominale $(87 \%)$. La cholangiopancréatographie rétrograde par voie endoscopique (CPRE) apparaissait csscnticlle pour des raisons diagnostiques : elle confirmait dans l'ensemble des cas l'existence d'une cholangite avcc ou sans stćnose papillairc*, ćliminait les rares diagnostics de sarcome de Kaposi ou de lymphome de la voic biliaire principale, et permettait des prćlèvements de bile à visée microbiologique et la réalisation d'unc sphinctérotomie endoscopique. Des anomalies

* Rétrécissement inflammatoire de la partie dislale de la biologiques ou morphologiques pancréatiques étaient notées chez $25 \%$ des patients.

Le rendement des examens microbiologiques était d'autant plus élevé que les prélèvements étaient multiples (biopsies duodénales, papillaires et prélèvement de bile). Les microorganismes retrouvés incluaient le Cryptosporidium (57\%), le cytomégalovirus $(28 \%)$ et une microsporidiose chez un patient $(7 \%)$. La survie à 12 mois après le diagnostic de cholangite était seulement de $14 \%$, ct les décès étaient rapportés à la progression du SIDA et non à la pathologie biliovésiculaire. La sphinctérotomie endoscopique permettait l'amćlioration, voire la disparition des douleurs abdominales chez $86 \%$ des patients. Les traitements spécifiques dirigés contre la cryptosporidiosc ou lc cytomégalovirus ne semblaient pas efficaces.

La cholangiopathic du SIDA apparaissait donc principalement d'originc infectieuse : des micro-organismes potentiellement responsables étaient identifiés chez 60 à $70 \%$ des patients. Le but second de notre travail a été d'évaluer prospectivement la cause des cholangiopathies associées à l'infection $\mathrm{VIH}$ chez les paticnts pour lesquels aucune infection opportuniste n'était identifiée. Les trois premières descriptions d'infection biliaire à Enterocytozoon bieneusi suggéraient que la microsporidiose pouvait êtrc l'une des causes des cholangiopathies du SIDA [5-7], comme cela avait été rapporté pour les diarrhées chroniques [8-9]. Les microsporidies sont des protozaires, parasites intracellulaires obligatoircs dont le cycle se décompose en deux phases, l'une proliférative mérogonique, l'autre de développement sporogonique à l'origine de sporoblastes, puis de spores. Dans notre étude [10], huit patients homosexuels infectés par le VIH (six du groupe IV de la classification du Center for Disease Control and Prevention et deux du groupe II asymptomatique avec des CD4 inféricurs à $10 / \mathrm{mm}^{3}$ ) ont été étudiés entre avril 1990 et septembre 1991 pour une cholangiopathic dont la cause n'avait pas été trouvée par les examens usuels. Tous les patients ont eu une échographie abdominale et une échoendoscopie ou unc CPRE autorisant un prélèvement $\mathrm{dc}$ bile dans la voie biliaire principale. Un paticnt a eu un prélèvement de bile par cathćtćrisme trans-hépatique et dcux autres au cours d'une cholćcystectomie pour cholécystite alithiasique. Les prélèvements de bile, les biopsies duodénales, papillaires, hépatiques ou le tissu vésiculaire ont étć étudiés cn microscopic optique et, pour certains, cn microscopie électroniquc.

Les huits patients, considérés commc ayant unc cholangiopathie inexpliquée du SIDA, avaicnt une microsporidiose biliaire. Des spores intra-épithéliales d'Enterocytozoon bieneusi (1 à $2 \mu \mathrm{m}$ ) et des plasmodiums supranucléaires étaicnt identifiés dans les six prélèvements duodénaux. La coloration au Gicmsa des prélèvements biliaires révélait des formes libres de microsporidies chez les huits patients et la microscopie élcctronique confirmait qu'il s'agissait d'Enterocytozoon bieneusi.

E. bieneusi était aussi présent dans les ccllules biliaires ductulaires d'une biopsie hépatique et dans le tissu vésiculaire des deux patients cholécystectomisés. Quatre patients avaient une cryptosporidiose associée, mais non détectée antérieurement, biliaire ou duodénalc ; un autre patient avait une infection associée par cytomégalovirus et Mycobacterium avium intra-cellulare, diagnostiquée par la biopsie hépatique. Depuis ce travail, nous avons observé six cas supplémentaires de cholangiopathie inexpliquée du SIDA : une microsporidiose biliaire était observée chez cinq patients et une mycobactériose biliaire chez le sixième. Les cholangiopathies du SIDA inexpliquécs par une infection à cryptosporidies ou à cytomégalovirus sont, probablement, principalement imputables à une infection à microsporidies qui apparaît 
comme la deuxième cause en fréquence de ces cholangiopathies.

\section{En conclusion}

Le diagnostic de cholangiopathie du SIDA est fondé sur des éléments simples cliniques (douleurs abdominales, fébricule, diarrhée chronique), biologiques (cholestase anictérique) et morphologiques (dilatation des voies biliaires intra- et/ou extra-hépatiques avec irrégularités pariétales, sténose papillaire). Il s'agit d'une complication souvent tardive, mais parfois révélatrice, de l'infection VIH chez des patients homosexuels ayant des fonctions immunitaires effondrées. Son étiologie est principalement infectieuse liée à des infections, parfois associées, à cryptosporidies, microsporidies et cytomégalovirus. La sphinctérotomie endoscopique apparaît comme un traitement de choix en cas de syndrome hyperalgique ou hyperfébrile, et l'intérêt du traitement spécifique mériterait d'être évalué précocement, avant la constitution de lésions fibrosantes irréversibles. Les progrès futurs dans l'approche de la cholangiopathie du SIDA doivent intéresser: (1) la thérapeutique avec des traitements plus précoces et plus efficaces contre les parasites ou virus responsables, si possible au stade de diarrhée chronique, avant la constitution des lésions cholangitiques ; (2) la compréhension de la physiopathogénie de cette complication: sa fréquence chez les homosexuels suggère une physiopathogénie liée aux habitudes sexuelles, comme cela a été montré pour le sarcome de Kaposi. Des modèles expérimentaux, comme ceux développés chez la souris pour la physiopathogénie de la cholangite sclérosante primitive, devraient permettre de mieux aborder ces problèmes. Un lien entre le profil immunogénétique et le risque de développement d'une cholangiopathie ne semble pas clair dans notre série, à l'inverse de ce qui a été proposé pour la cholangite sclérosante primitive (HLA A1B8 DR3 DQ W52 A)

\footnotetext{
Stanislas Pol

Unité d'hépatologie, hôpital Laënnec, 42, nue de Sères, 75340 Paris Cedex 07, France.
}

$\mathrm{m} / \mathrm{s} \quad n^{\circ} \quad 6-7$ vol. 9, juin-juillet 93
RÉFÉRENCES

1. Pitlik SD, Fainstein V, Rios A, Guarda L, Mansell PWA, Hersh EM. Cryptosporidial cholecystitis (letter). N Engl J Med 1983 ; 308 : 967.

2. Cello JP. Acquired immunodeficiency syndrome cholangiopathy: spectrum of the discase. Am J Med $1989 ; 86: 539-46$.

3. Dowsett JJ, Davidson RMR, Vaira D, Polydorou A, Clairns SR, Weller IVD. Sclerosing cholangitis in acquired immunodeficiency syndrome. Scand J Gastroenterol 1988 ; $23: 1267-74$.

4. Bouche H, Housset C, Dumont JL, Carnot F, Menu Y, Aveline B, Belghiti J, Boboc B, Erlinger S, Berthelot P, Pol S. AIDSrelated cholangitis : features and course in 15 patients. J Hepatol $1993 ; 17$ : 34-9.

5. Mac Whinney PHM, Nathwani D, Green ST, Boyd JF, Forrest JAH. Microsporidiosis detected in association with AIIDS-related sclerosing cholangitis. AIDS 1991; 5 : 1394-5.

6. Pol S, Romana C, Richard S, Carnot F, Dumont JL, Bouche H, Pialoux G, Stern M, Pays JF, Berthelot P. Enterocytozoon bieneusi infection in acquired immunodeficiency syndrome-related cholangitis. Gastroenterology $1992 ; 102$ : 1778-81.

7. Beaugerie L, Teilhac MF, Delmol AM, Fritsch J, Girard PM, Rozenbaum W, Le Quintrec Y, Chatelet PF. Cholangiopathy associated with Microsporidia infection of the common bile duct mucosa in a patient with HIV infection. Ann Int Med 1992; 117 401-2.

8. Desportes I, Le Charpentier Y, Galian A, Bernard F, Cochand-Priollet B, Lavergne A Ravisse P, Modigliani R. Occurrence of a new microsporidian : Enterozytozoon bieneusi $\mathrm{n}$ g., n. sp., in the enterocytes of a human patient with AIDS. J Protozool 1985; 32 : 250-4.

9. Orenstein JM, Chiang J, Steinberg W, Smith PD, Rotterdam $H$, Kolter DP. Intestinal microsporidiosis as a cause of diarrhea in human immunodeficiency virus-infected patients : a report of 20 cases. Hum Pathol $1990 ; 21: 475-81$.

10. Pol S, Romana C, Richard S, Amouyal P, Desportes-Livage I, Carnot F, Pays JF, Berthelot P. Microsporidia infection in patients with the human immunodeficiency virus and unexplained cholangitis. N Engl J Med 1993 ; $328: 95-9$

\section{TIRÉS A PART}

\section{S. Pol.}

